Signal \& Image Processing : An International Journal (SIPIJ) Vol.6, No.3, June 2015

\title{
A STUDY OF SPECKLE NOISE REDUCTION FILTERS
}

\author{
Jyoti Jaybhay and Rajveer Shastri \\ Department of Electronics Engineering, \\ VPCOE Baramati , Pune University, India
}

\begin{abstract}
Ultrasound images and SAR i.e. synthetic aperture radar images are usually corrupted because of speckle noise also called as granular noise. It is quite a tedious task to remove such noise and analyze those corrupted images. Till now many researchers worked to remove speckle noise using frequency domain methods, temporal methods, and adaptive methods. Different filters have been developed as Mean and Median filters, Statistic Lee filter, Statistic Kuan filter, Frost filter, Srad filter. This paper reviews filters used to remove speckle noise.
\end{abstract}

\section{KEYWORDS}

Speckle Noise, Multiplicative noise, Ultrasound images, Scalar filters, Adaptive filters.

\section{INTRODUCTION}

Human beings are suffering from very dangerous and long term diseases as Cancer, Blood Processing, Heart blockage, Kidney failure, Asthma etc. Detection of such diseases at very early stage is very necessary thing. Also, it should be real time. Image processing as ultrasound imaging plays an important role in this task. Now a day it has become easy, real-time, low cost and also it has non-invasive nature. Still removal of noise from ultrasound imaging is very challenging task [1].

Scattering and reflection [2] are two major phenomena in medical ultrasound imaging which are closely related concepts. When particles are larger than the wavelength causes reflection and scattering occurs when the scatterers are smaller than the sound's wavelength. When the backscattered acoustic pulses received are in phase or out of phase, this phenomenon leads to both constructive and destructive interference and appears as a granular pattern called speckle noise [3]. Speckle noise affects the quality of ultrasound image and it also reduces important information from image as edge, shape, intensity value etc. Corrupted ultrasound images contain high frequency components [4] and to remove them filters are used. Many researches are made till now to despeckle the image like image processing filters.

This paper gives information on methods used to removal of noise from echo images. This work is arranged in this paper as per following. Section II describes speckle model. Section III reviews 
Signal \& Image Processing : An International Journal (SIPIJ) Vol.6, No.3, June 2015

the different filters used for speckle noise reduction as 1.Scalar filters and 2.Adaptive filters. Section IV reviews performance metrics and section V concludes the review.

\section{SPECKLE MODEL}

Speckle noise [5] is the characteristic effect seen in ultrasound images that contribute to the visual noise. The image of a relatively uniform object with many scattering sources within a resolution cell will have pixel values that vary randomly with position due to constructive and destructive interference. Ultrasound images mostly get corrupted because of speckle noise. It is multiplicative noise, having granular pattern. Mathematically Speckle noise [6] is expressed as in eqn. (1)

$$
g(m, n)=f(m, n) * u(m, n)+\eta(m, n)
$$

Where $g(m, n)$ is corrupted image, $u(m, n)$ is multiplicative component and $\eta(m, n)$ is additive component. For ultrasound images, it is necessary to remove additive noise but multiplicative can be allowed, given in eqn. (2)

$$
\begin{aligned}
& g(m,)=f(m, n) * u(m, n)+\eta(m, n)-\eta(m, n) \\
& (m, n)=f(m, n) * u(m, n)
\end{aligned}
$$

Also, speckle noise follows gamma distribution [7] which is shown below

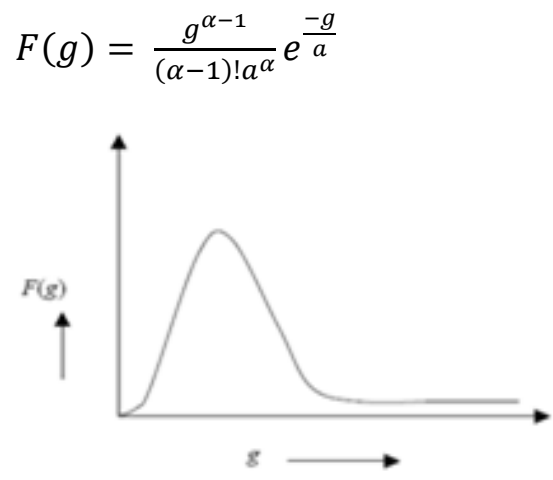

Figure 1. Gamma Distribution

Here $a^{\alpha}$ is variance and $\mathrm{g}$ is gray level. Gamma distribution is represented as in Fig. 1. Speckle noise has following characteristics [7]:

1. Speckle noise is a multiplicative noise which is in direct proportion to the local gray level in any area.

2. The signal and the noise are statistically independent.

3. The sample mean and variance of a single pixel are equal to the mean and variance of the local area. 
Signal \& Image Processing : An International Journal (SIPIJ) Vol.6, No.3, June 2015

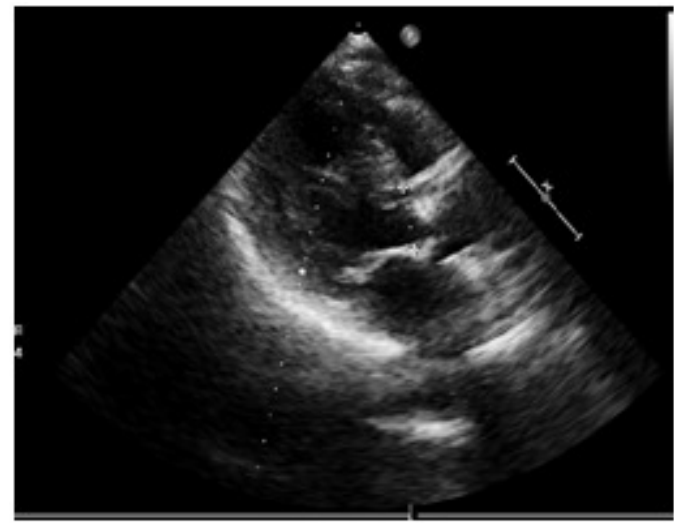

Figure 2. Original Image

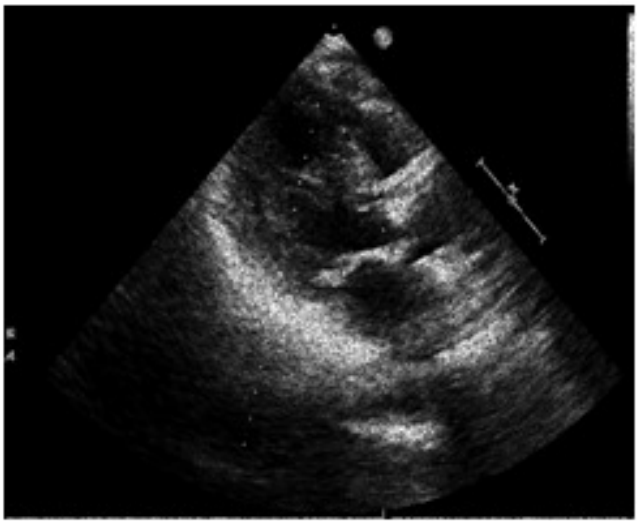

Figure 3. Image with Speckle Noise

\section{SPECKLE REDUCTION FILTERS}

There are different types of noise that appears in images. Noise may occur due to different factors such as while acquiring images, capturing images, transforming images, compressing images etc. Noise may have different types, and hence it is necessary to provide different techniques according to the type of noise. Speckle noise contains high frequency components due to temporal movement of organs as brain, heart etc. So it is necessary to provide low pass filter to remove the high frequency noise. To remove speckle noise from images till now many filters are used [8]. Some filters are good in visual interpretation where as some are good in smoothing capabilities and noise reduction. Some examples of such filters are Mean, Median, Lee, Kuan, Frost, Enhanced Frost, Wiener and Gamma MAP filters [9]. Some of these use window technique to remove speckle noise, called as kernel [10]. This window size can range from 3-by-3 to 33-by33 but it must be odd. To achieve better result window size should be smaller.

\subsection{Scalar Filters}

Scalar filters [11] are based on the ratio of local statistics, which improves smoothing in homogenous regions of the images where speckle is fully developed and reduces appreciably in the other regions of the image in order to preserve the useful details of the image. Fundamental types of scalar filters are Mean filter and Median filters discussed in next sections.

\subsubsection{Mean Filter [12]}

It is simple and intuitive filter invented by Pomalaza-Raez in 1984. It does not remove speckle noise at whole but reduces at some extend. It works on average basis that is the centre pixel is replaced by the average of the all pixels. Hence this filter gives blurring effect to the images, so it is least satisfactory method to remove speckle noise as it results in loss of details. Mathematical representation is given in eqn. (4) for $m \times n$ window region.

$$
h(i, j)=\frac{1}{m n} \sum_{k \in m} \sum_{l \in n} f(k, l)
$$


Signal \& Image Processing : An International Journal (SIPIJ) Vol.6, No.3, June 2015

\subsubsection{Median Filter [13]}

It is non linear filter invented by Pitas in 1990. It gives quite better result than the mean filter. Here center pixel is replaced by the median value of all pixels and hence produces less blurring. Due to this nature it is used to reduce impulsive speckle noise. Advantage is it preserves the edges. Disadvantage is extra time needed for computation of the median value for sorting $\mathrm{N}$ pixels, the temporal complexity is $\mathrm{O}(\mathrm{N} \log \mathrm{N})$. Median filter follows algorithm as follows:

1. Take a $3 \times 3$ (or $5 \times 5$ etc.) region centered around the pixel $(i, j)$.

2. Sort the intensity values of the pixels in the region into ascending order

3. Select the middle value as the new value of pixel $(i, j)$.

\subsection{Adaptive Filters}

Several adaptation methods are proposed to achieve a better result by varying window size [14] and also to preserve the features like edges. Filters that are having adaptive nature are discussed in following sections:

\subsubsection{Frost filter [15]}

Invented by Frost in 1982, is linear, convolutional filter used to remove the multiplicative noise from images. As compared to mean and median filter it has adaptive nature and also it is exponentially-weighted averaging filter. Frost filter works on the basis of coefficient of variation which is the ratio of local standard deviation to the local mean of the corrupted image. Within the kernel size of n-by-n then the centre pixel value is replaced by weighted sum of values of the neighbourhood in kernel. The weighting factor decrease as we go away from interested pixel and increase with variance. It assumes multiplicative noise. Frost filter follows formula given by eqn. (4).

Where,

$$
D N=\sum_{n \times n} K \alpha e^{-\alpha|t|}
$$

$$
\begin{aligned}
& \quad \alpha=\left(\frac{4}{n \overline{\sigma^{2}}}\right)\left(\frac{\sigma^{2}}{\overline{I^{2}}}\right) \\
& \mathrm{K}=\text { Normalized constant } \\
& \bar{I}=\text { Local Mean } \\
& \sigma=\text { Local variance } \\
& \bar{\sigma}=\text { Image coefficient of variation value } \\
& |\mathrm{t}|=\left|\mathrm{X}-X_{0}\right|+\left|\mathrm{Y}-Y_{0}\right| \\
& \mathrm{n}=\text { moving kernel size }
\end{aligned}
$$

\subsubsection{Lee Filter}

It is developed by Jong Sen Lee in 1981 [16]. It is better than above filters in edge preservation. It is based on multiplicative speckle model and uses local statistics to preserve details. Lee filter works on the variance basis, i.e. if variance of the area is low then it performs smoothing operation but not for high variance. That means it can preserve details in low as well as in high contrast hence it has adaptive nature. Mathematical model for Lee filter is given in eqn. (5):

$$
\operatorname{Img}(i, j)=\operatorname{Im}+W^{*}(C p-\operatorname{Im})
$$


Signal \& Image Processing : An International Journal (SIPIJ) Vol.6, No.3, June 2015

Where, Img - pixel value after filtering

Im - mean intensity of filter window

$C p$ - Center pixel

$W$-filter window, $W=\sigma^{2}\left(\sigma^{2}+\rho^{2}\right)$

$\sigma^{2}$ is the variance of the pixel calculated as

$\sigma^{2}=\left[1 / N \sum_{j=0}^{N-1}\left(X_{j}\right)^{2}\right]$

$N=$ size of filter window, $X_{j}=$ pixel value at $\mathrm{j}$.

$\rho=$ additive noise variance, for $\mathrm{M}$ size of image and $Y_{i}$ value of each pixel it is given as:

$\rho^{2}=\left[1 / M \sum_{j=0}^{M-1}\left(Y_{j}\right)^{2}\right]$

For no smoothing filter output is the only mean intensity value (Im). Disadvantage of Lee Filter is it cannot effectively remove the speckle noise near edges.

\subsubsection{Kuan filter [17]}

It was developed by Kuan and Nathan and Kurlander in 1987. It is local linear minimum mean square error filter under multiplicative noise. It is quite advanced than Lee filter in a factor as it has no approximation involved. It transforms the multiplicative speckle model into the additive linear form. Weighted function $\mathrm{W}$ is for Kuan filter is given by,

$$
W=\frac{\left(1-\frac{C u}{C i}\right)}{(1+C u)}
$$

Where,

$\mathrm{Cu}=$ estimated noise variation coefficient.

$C u=\sqrt{1 / E N L}, \mathrm{ENL}=$ equivalent noise looks.

$C i=$ variation coefficient of image.

$\mathrm{Ci}=\mathrm{S} / \mathrm{Im}$

$\mathrm{S}=$ standard deviation in the filter window.

\subsubsection{Enhanced Frost and Enhanced Lee Filter [18]}

Proposed by Lopes in 1990, works on the basis of the threshold value. Averaging is done when the local coefficient of variation below a lower threshold. Filter will perform strictly as all pass filter when local coefficient of variation is above the higher threshold. And when the local variance is in between both thresholds then balance between averaging and identity operation is done.

\subsubsection{Gamma Map Filter [19]}

Proposed by Lopes in 1993, uses coefficient variation and contrast variation. It is again better than Frost and Lee filter and also minimizes the loss of texture information. Working of Gamma Map filter is similar to Enhanced Frost filter except that if local coefficient of variation falls between two thresholds then pixel value is based on the Gamma estimation of the contrast ratios. 


\subsubsection{Wiener Filter [20]}

It was proposed by Norbert Wiener during the 1940s and published in 1949. It is also known as Least Mean Square Filter. It has capacity to restore images even if they are corrupted or blurred. It reduces noise from image by comparing desired noiseless image. Wiener filter works on the basis of computation of local image variance. Hence when local variance of the image is large the smoothing is done in lesser amount and if local variance is small it performs more smoothing. This approach of Wiener filter results better than linear filtering [13]. Wiener filter requires more computation time. It has following mathematical formula:

$$
f(u, v)=\left[\frac{H(u, v)^{*}}{H(u, v)^{2}+\left[\frac{S n(u, v)}{S f(u \cdot v)}\right]}\right] G(u, v)
$$

Where,

$H(u, v)=$ Degradation function and

$H(u, v)^{*}=$ conjugate complex

$G(u, v)=$ Degraded image

$\operatorname{Sn}(u, v)=$ Power spectra of noise.

$S f(u, v)=$ Power spectra of original image.

\section{Performance analysis Parameters}

To analyze the speckle reduction methods various performance parameters are used and compared such as Signal-to-noise ratio (SNR), root mean square error (RMSE), mean absolute error (MAE), peak signal-to-noise ratio (PSNR), average peak signal-to-noise ratio (APSNR), Pratt's figure of merit (FoM), contrast-to-noise ratio (CNR), structural similarity (SSIM), Edgeregion mean square error (MSE), new quality index (NQI), video quality metric (VQM). These are described in the following sections.

\subsection{SNR [21]}

Parameter is a basic measure metric that is used to measure the level of noise as a ratio of the mean $\sigma_{g}{ }^{2}$ to the standard deviation $\sigma_{e}{ }^{2}$ of pixel value in an image. SNR has theoretical value 1.91 for a fully developed speckle. It has indirect proportion with speckle. It has higher value for lower speckle.

\subsection{RMSE [22]}

$$
S N R=10 \log _{10} \frac{\sigma_{g}^{2}}{\sigma_{e}^{2}}
$$

It stands for root mean square error. It measures the average squared difference between the original and filtered images, where the original and filtered images have size $X \times Y$ pixels. Accordingly, the RSME is the root of MSE given in equations (10) and (11).

$$
\begin{gathered}
M S E\left(I_{\text {filt }} I_{\text {ref }}\right)=\frac{1}{X Y} \sum_{i=1}^{Y} \sum_{j=1}^{X}\left(I_{\text {filt }}(i, j)-I_{\text {ref }}(i, j)\right)^{2} \\
R M S E=\sqrt{M S E}
\end{gathered}
$$


Signal \& Image Processing : An International Journal (SIPIJ) Vol.6, No.3, June 2015

\subsection{PSNR and APSNR [23]}

PSNR is defined from RMSE. It is the ratio between the possible power of a signal and the power of corrupting noise. For 256 gray levels, PSNR is defined as

$$
P S N R=20 \log _{10}\left(\frac{255}{R M S E}\right)
$$

A simple average of PSNR per frame is called APSNR.

\subsection{MAE [24]}

It is the mean absolute error between two images being compared.

$$
\operatorname{MAE}\left(I_{\text {filt }}, I_{\text {ref }}\right)=\frac{1}{X Y} \sum_{i=1}^{Y} \sum_{j=1}^{X}\left|I_{\text {filt }}(i, j)-I_{\text {ref }}(i, j)\right|
$$

\subsection{Pratt's FoM [14]}

This parameter is an estimator for quantifying the edge pixel displacement between the edge masks of filtered and reference images, and is defined as

$$
\operatorname{FoM}\left(I_{\text {filt }}, I_{\text {ref }}\right)=\frac{1}{\max \left(N_{\text {filt }}, N_{\text {ref }}\right)} \sum_{i=1}^{N} \frac{1}{1+d_{i}^{2} \alpha}
$$

Where, pixel, and

$d i=$ Euclidean distance between the $i$ th detected edge pixel and the nearest original edge

$$
\alpha=\text { constant and set to } 0.11
$$

\subsection{CNR [15]}

This metric operates on a single image and exploits levels of contrast between two different regions of images. One region is a region of interest (ROI) and the other can be a part of the background. This metric is calculated as

$$
\mathrm{CNR}=\frac{|\mu 1-\mu 2|}{\sqrt{\sigma 1^{2}}+\sigma 2^{2}}
$$

Where, $\mu 1$ and $\sigma 1$ are mean and variance of ROI and $\mu 2$ and $\sigma 2$ are mean and variance of background.

\subsection{SSIM [1]}

This index is another metric for measuring the similarity between two images. This metric has much better consistency with the qualitative appearance of the image.

$$
S S I M=\frac{1}{M} \sum \frac{(2 \mu 1 \mu 2+C 1)\left(2 \sigma_{1,2}+C_{2}\right)}{\left(\mu_{1}^{2}+\mu_{2}{ }^{2}+C_{1}\right)\left(\sigma_{1}{ }^{2}+\sigma_{2}{ }^{2}+C_{2}\right)}
$$


Signal \& Image Processing : An International Journal (SIPIJ) Vol.6, No.3, June 2015

Where, $\mu 1$ and $\mu 2$ are the means and $\sigma 1$ and $\sigma 2$ are the standard deviations of the images being compared. $\sigma_{1,2}$ is the covariance between them. SSIM has value between 0 and 1 , when it is equal to 1 images are structurally equal.

\subsection{Edge-Region MSE [1]}

It measures the average absolute difference between two images:

$$
\operatorname{MSE}\left(I E_{f i l t}, I E_{r e f}\right)=\frac{1}{X Y} \sum_{i=1}^{Y} \sum_{j=1}^{X}\left(I E_{f i l t}(i, j)-I E_{r e f}(i, j)\right)^{2}
$$

Where $I E_{\text {filt }}$ and $I E_{\text {ref }}$ are edges of filtered and reference images respectively. The edge-region MSE measures the average differences in edge regions.

\subsection{NQI [1]}

It is video quality metric is first measured frame by frame. For each frame, the distortion is modelled as a combination of three different factors: loss of correlation, luminance distortion, and contrast distortion. This metric is calculated as

$$
Q=\frac{4 \sigma_{x y} \overline{x y}}{\left(\sigma_{x}^{2}+\sigma_{y}^{2}\right)\left[(\bar{x})^{2}+(\bar{y})^{2}\right]}
$$

\subsection{VQM [1]}

This metric is a modified discrete cosine transform (DCT)-based video quality evaluation measure that is based on the spatial-temporal property of human visual perception. The result of this metric is comparable with RMSE-based metrics and performs much better in situations in which RMSE fails.

\section{CONCLUSIONS}

Speckle reduction filters such as Scalar filters and Adaptive filters are described in this paper. Filtering methods using scalar filters are good for removing high frequency noise but fail to preserve the edge details. Whereas Adaptive filter methods are more successful than filtering as it preserves the details. Also, adaptive filter method requires the more computation time. This drawback can be overcome by proper selection of weighting function.

\section{REFERENCES}

[1] P. Gifani, H. Behnam, Zahra Alizadeh Sani, "Noise Reduction of Echocardiographic Images Based on Temporal Information" IEEE Trans. on Ultrasonics, Ferroelectrics, and Frequency Control, vol. 61 no. 4, Apr. 2014.

[2] J. Fredrik Synnevag Carl-Inge Colombo Nilsen, "Speckle Statistics in Adaptive Beamforming" Department of Informatics, University of Oslo, N-0316 Oslo, Norway.

[3] A. Achim, A. Bezerianos P. Tsakalides," Novel Bayesian Multiscale Method for Speckle Removal in Medical Ultrasound Images" IEEE Trans. On Medical Imaging, vol. 20, no. 8, Aug. 2001. 
Signal \& Image Processing : An International Journal (SIPIJ) Vol.6, No.3, June 2015

[4] R. Wagner, Smith, John M. Sandrik and Lopez, "Statistics of Speckle in Ultrasound B-Scans" IEEE Trans. on Sonics and Ultrasonics, vol. 30, no. 3, May 1983.

[5] S. Karabchevsky, David Kahana, Ortal Ben-Harush, and Hugo Guterman, "FPGA-Based Adaptive Speckle Suppression Filter for Underwater Imaging Sonar" IEEE Journal Of Oceanic Engg., vol. 36, no. 4 , Oct. 2011.

[6] M. Sarode, Prashant R. Deshmukh, "Reduction of Speckle Noise and Image Enhancement of Images Using Filtering Technique" International Journal of Advancements in Technology vol 2, no. 1 Jan. 2011.

[7] R. Garg, Er. Abhijeet Kumar, "Comparison of Various Noise Removals Using Bayesian Framework" International Journal of Modern Engineering Research vol.2, Issue.1, Jan-Feb 2012 pp-265-270.

[8] T. Joel and R. Sivakumar, “ Despeckling of Ultrasound Medical Images: A Survey” Journal of Image and Graphics vol. 1, no. 3,Sept. 2013

[9] C.I. Christodouloul, C. Loizou, C.S. Pattichis, M. Pantziarisl, E. Kyriakoul, M.S. Pattichis, C.N. Schizas, A. Nicolaides, "De-speckle Filtering in Ultrasound Imaging of the Carotid Artery" Proceedings of the Second Joint EMBSiBMES Conference Houston, TX, USA Oct. 23-26.2002 IEEE.

[10] O. Lopera, R. Heremans, A. Pizurica, Yves Dupont, "Filtering speckle noise in SAS images to improve detection and identification of seafloor targets"

[11] P. Courmontagne, IEEE IM2NP / ISEN-Toulon, France, "Speckle noise reduction: a review Advances in Seafloor-Mapping Sonar" Dec. 1st, Brest

[12] R. C. Gonzalez, Woods, R. E., 2002. Digital Image Processing, Addison-Wesley Inc.

[13] T. Huang, George J. Yang and Gregory Y. Tang, "A Fast Two Dimensional Median Filtering Algorithm” IEEE Trans. on Acoustics, Speech and signal processing, vol. ASSP-27, no. 1 Feb. 1979.

[14] D. Donoho, "De-Noising by Soft-Thresholding" IEEE Trans. on Information Theory, vol. 41, no. 3, May 1995.

[15] Y. Yu, Scott T. Acton, "Speckle Reducing Anisotropic Diffusion" IEEE Trans. On Image Processing , vol. 11. Nov. 2002.

[16] J. Lee, "Digital Image Enhancement and Noise Filtering By Use Of Local Statistics" IEEE Trans. On Pattern Analysis and Machine Intelligence, vol. Pami-2, no.-2 March 1980.

[17] S.Kalaivani Narayanan and R.S.D.Wahidabanu, "A View on Despeckling in Ultrasound Imaging" International Journal of Signal Processing,Image Processing and Pattern Recognition vol. 2, no.3, Sept. 2009

[18] Y. Huang, J. L. van Genderen, "Evaluation of Several Speckle Filtering techniques for ERS-1\&2 Imagery" International Archives of Photogrammetry and Remote sensing. vol. XXXI, Part B2. Vienna 1996.

[19] D. A. Nelson Mascarenhas, "An Overview of Speckle noise filtering in SAR images." European Space agency. Provided by the NASA Astrophysics Data System. 
Signal \& Image Processing : An International Journal (SIPIJ) Vol.6, No.3, June 2015

[20] A. Garg, Jyoti Goal, Sandeep Malik, Kavita Choudhary, Deepika, "De-speckling of Medical Ultrasound Images using Wiener Filter and Wavelet Transform" International Journal of Electronics \& Comm. Technology vol. 2, Issue 3, Sept. 2011.

[21] R. Czerwinski, Douglas L. Jones, and William D. O'Brien, "Detection of Lines and Boundaries Speckle Images Application to Medical Ultrasound" IEEE Trans. on Medical Imaging, vol, 18 no. 2,Feb. 1999

[22] K. Bala Prakash, R. Venu Babu, B. Venu Gopal,"Image Independent Filter for Removal of Speckle Noise”, International Journal of Computer Science Issues, vol. 8, Issue 5, no 3, Sept. 2011.

[23] K. Kaur, Baljit Singh and Mandeep Kaur, "Speckle Noise Reduction Using 2-D FFT In Ultrasound Images" International Journal of Advances in Engineering \& Technology, Sept. 2012. of Speckle Noise Reduction Filters On Active Radar And SAR Images."

[24] Li c "Two adaptive filters for speckle reduction in SAR imagery by using the variance ratio", International general of remote sensing vol. 9, no. 4, pp. 641-653. 1988. 